J. Lake Sci. (湖泊科学) , 2012, 24(6): 838-842

http: //www.jlakes.org. E-mail : jlakes@niglas.ac.cn

(c) 2012 by Journal of Lake Sciences

\title{
利用生物膜强化表流湿地处理农村生活污水的试验”
}

\author{
张云慧 ${ }^{1}$, 朱 伟 $^{1,2}$, 董 婵 ${ }^{2}$ \\ ( 1 : 河海大学环境学院,南京 210098$)$ \\ $(2$ :河海大学水资源高效利用与工程安全国家工程研究中心 , 南京 210098)
}

\begin{abstract}
摘 要: 针对农村生活污水难以集中处理的问题, 提出了采用生物膜强化表流湿地工艺就地进行农村生活污水处理的方 法, 并对其进行试验研究. 结果表明: 在表流湿地表面设置生物膜载体后, 系统的水质净化能力提高, 处理负荷较高的条 件下 $\left(\mathrm{COD}_{\mathrm{Cr}}: 657.27 \mathrm{mg} / \mathrm{L} 、 \mathrm{TN}: 26.27 \mathrm{mg} / \mathrm{L} 、 \mathrm{TP}: 8.66 \mathrm{mg} / \mathrm{L}\right.$ 和 $\mathrm{SS}: 276 \mathrm{mg} / \mathrm{L}$ ), 处理效果显著提高. 在效果明显的试验组 中, 生物膜设置后系统对 $\mathrm{TN} 、 \mathrm{TP} 、 \mathrm{COD}_{\mathrm{Cr}}$ 的去除率分别提高了 $21.64 \% 、 16.24 \% 、 19.12 \%$. 另外, 通过对系统中微生物的检 测发现, 生物膜载体的引入对湿地系统本身影响不大, 系统水质净化能力的提高主要源于载体上微生物的增加. 该方法 在未增加系统占地面积的情况下提高了水质净化能力,为解决农村生活污水处理难的问题提供了新的思路.
\end{abstract}

关键词: 表流湿地; 生物膜;农村生活污水;功能强化

\section{Treatment for rural domestic sewage with biofilm aggrandizement in surface flow con- structed wetland}

ZHANG Yunhui ${ }^{1}$, ZHU Wei ${ }^{1,2} \&$ DONG Chan ${ }^{2}$

(1: College of Environment, Hohai University, Nanjing 210098, P. R. China)

(2: National Engineering Research Center of Water Resources Efficient Utilization and Engineering Safety, Hohai University, Nanjing 210098, P. R. China)

\begin{abstract}
Aiming to the problem of the difficult central treatment on rural domestic sewage, a new approach of biofilm aggrandizement on the treatment of rural domestic sewage on the spots in surface flow constructed wetland was raised and corresponding experiments were carried out. Results showed that water purification ability improved obviously after setting the biofilm carrier in surface flow constructed wetland, especially under higher pollution load $\left(\mathrm{COD}_{\mathrm{Cr}}: 657.27 \mathrm{mg} / \mathrm{L}\right.$, TN: $26.27 \mathrm{mg} / \mathrm{L}, \mathrm{TP}: 8.66 \mathrm{mg} / \mathrm{L}$, SS : $276 \mathrm{mg} / \mathrm{L})$. The removal rates of TN, TP and $\mathrm{COD}_{\mathrm{Cr}}$ were increased by $21.64 \%, 16.24 \%$ and $19.12 \%$, respectively. In addition, the setting of the biofilm carrier had little impacts on the wetland system itself through the detection of microorganisms in wetland. The improvement of the water purification ability mainly resulted from the increase of the microorganism on the biomass carrier. This new method could improve the wetland efficacy without expanding wetland areas, which provides a promising solution for rural domestic sewage.
\end{abstract}

Keywords: Surface flow constructed wetland; biofilm; rural domestic sewage; enriched purification function

农村生活污水是我国的主要污染来源之一 ${ }^{[1]}$. 至 2010 年底, 我国有 858 个县城没有可投人运行的污水 处理厂,96\% 的村庄没有排水管道和污水处理系统. 农村每年产生的约 80 多亿吨生活污水随意排放 ${ }^{[2]}$. 由 于农村生活污水较为分散, 收集比较困难, 为了避免对周围环境造成污染, 就地处理显得尤为必要. 人工湿 地是一种适合农村的就地处理方法, 由于处理效果较好, 在生活污水处理中已有一些应用 ${ }^{\left[{ }^{[3]}\right.}$. 但由于潜流湿 地存在后续维护管理复杂以及出现滤料堵塞的问题 ${ }^{[4-5]}$, 影响了这一技术在农村的广泛应用. 相对而言, 表 流人工湿地对后续维护管理的要求相对较低, 布水系统的动力要求较小, 植物的轮作、更替也比较容易, 尤

* 国家重点基础研究发展计划 “973” 项目 (2012CB719804)、江苏省重点基金项目 (SBK201150024) 和国家自然科学 基金项目 (50979028) 联合资助. 2012-02-14 收稿;2012-04-12 收修改稿. 张云慧,女, 1985 年生,硕士研究生; E-mail:zyhyun@126. com. 
其是不存在堵塞的问题, 该技术似乎更加适合在农村大面积推广. 然而, 相对于潜流湿地而言, 表流湿地的 单位面积净化效率明显偏低 ${ }^{[6]}$, 在可利用面积偏小、生活污水浓度较高时, 往往难以满足处理的要求 ${ }^{[7]}$, 因 此如何提高表流人工湿地的水质净化能力成为值得探讨的问题.

表流湿地对较低浓度的污水具有较好的净化效果 ${ }^{[8]}$, 但对于高浓度污水却难以达到净化的要求. 陈俊 敏等 ${ }^{[9]}$ 利用 $400 \mathrm{~m}^{2}$ 人工芦苇湿地对农家乐旅游设施污水 $\left(\mathrm{COD}_{\mathrm{Cr}}\right.$ 为 $123 \sim 180 \mathrm{mg} / \mathrm{L}$ 、氨氮为 $51 \sim 82 \mathrm{mg} / \mathrm{L}$ 、悬 浮质 SS 为 $34 \sim 108 \mathrm{mg} / \mathrm{L}$ ) 进行处理, 在布水速率 $2.77 \mathrm{~cm} / \mathrm{d}$ 的情况下, 去除率分别仅达到 $47.97 \%$ 、 $76.19 \% 、 30.77 \%$, 难以达到允许排放的标准. 表流湿地对于悬浮态污染物的截留作用比较明显, 但对于溶 解态的污染物, 作用受到限制.

湿地中大部分溶解性污染物质的去除主要依靠微生物的分解 ${ }^{[10]}$, 其分解的污染物通常占整个系统污染 物去除的 $80 \%$ 左右 ${ }^{[11]}$. 表流湿地系统中 $81 \%$ 95\% 的微生物位于基质土壤中,与污染物的接触时间、接触面 积受到其水流特点的影响 ${ }^{[12]}$. 付融冰等 ${ }^{[13]}$ 对湿地基质中微生物的数量及其与水质净化效果之间的关系进 行分析, 表明随着基质深度、距进水口距离增加, 微生物的数量逐渐降低; 基质中微生物数量与 $\mathrm{BOD}_{5}$ $(r=0.897, P<0.05)$ 及总氮 $(\mathrm{TN})(r=0.988, P<0.01)$ 去除率之间的相关性显著. 因此, 可通过增加系统 中的微生物量提高湿地系统的水质净化能力.

在自由表面垂直流人工湿地中, Iamchaturapatr ${ }^{[14]}$ 通过种植不同植物来改变与水的接触面进而影响微生 物量, 探讨增加接触面来提高净化效果的方法. 从原理上分析, 要提高湿地的净化能力, 需从提高湿地系统 中的微生物量以及微生物与污染物接触面积这两个方面进行考虑. 因此通过上述启发, 提出在表流湿地表 面设置生物膜,达到强化表流湿地净化农村生活污水的效果.

\section{1 材料与方法}

\section{1 试验污水}

试验所用污水根据农村生活污水特 点人工配制而成. 配置方法如下: 取生活 污水处理厂中的污泥, 先用水溶解, 不断 搅拌后静置一段时间, 取上层清液作为试 验污水. 所配试验污水分为低浓度组和高 浓度组, 相应的指标分别为 $\mathrm{COD}_{\mathrm{Cr}}: 243.62 \pm$ $11.43 、 657.27 \pm 18.40 \mathrm{mg} / \mathrm{L} ; \mathrm{TN}: 14.46 \pm$ $0.26 、 26.27 \pm 0.69 \mathrm{mg} / \mathrm{L}$; 总磷 $(\mathrm{TP})$ : $2.69 \pm 0.05 、 8.66 \pm 0.42 \mathrm{mg} / \mathrm{L} ; \mathrm{SS}: 159 \pm$ $4.87 、 276 \pm 14.86 \mathrm{mg} / \mathrm{L}$.

\section{2 表流湿地试验模型}

在河海大学校园构建了两个表流湿地 模型. 模型长 $1.0 \mathrm{~m}$ 、宽 $0.8 \mathrm{~m}$ 、高 $0.6 \mathrm{~m}$ 、水 深 $0.20 \sim 0.25 \mathrm{~m}$. 采集南京长江边河滨湿地 土壤作为湿地土壤, 厚度为 $0.3 \sim 0.4 \mathrm{~m}$. 模 型湿地经过 4 个月运行形成了以香蒲为优 势种的湿生植物群落, 植株密度约 60 株 $/ \mathrm{m}^{2}$. 试验污水由进水管进人后, 经表流湿地后由 出水管流出进人循环水桶中, 再由水洜打人 进水管之中,通过循环布水满足设定水力负 荷的要求. 表流湿地试验模型见图 1.

\section{3 生物膜的种类和设置方法}

根据农村的实际情况采用农村常见 的玉米皮作为生物膜载体 (图 2). 将玉米
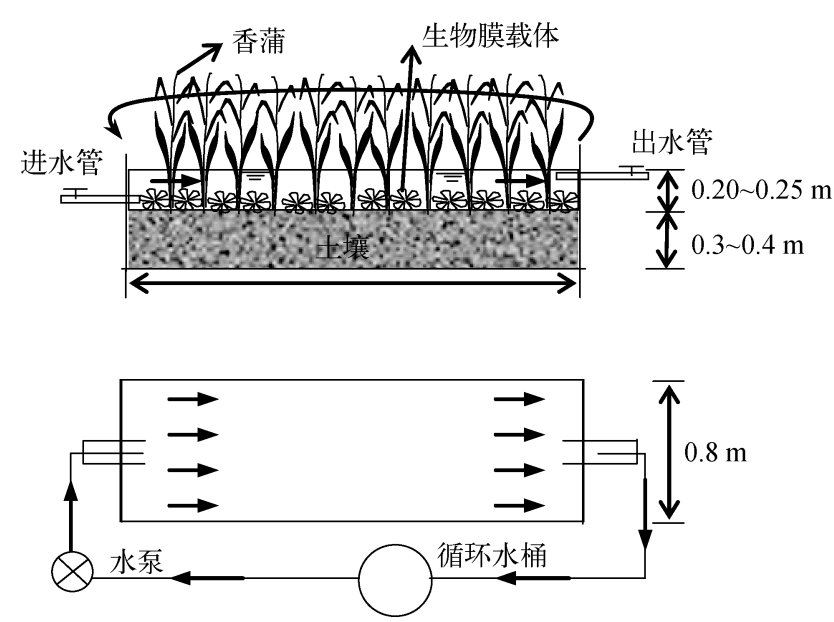

图 1 表流湿地试验系统

Fig. 1 The surface flow constructed wetland system

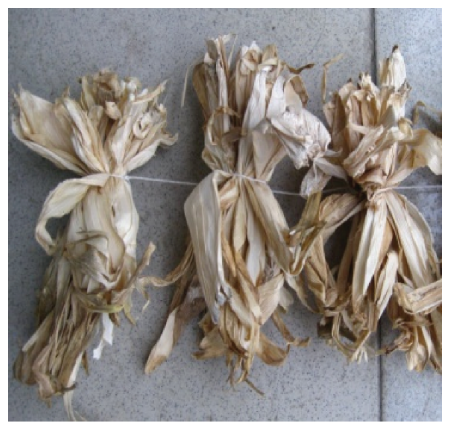

图 2 用玉米皮制作的生物膜载体

Fig. 2 The biofilm carrier by corn husk 
皮制作成直径为 $18 \mathrm{~cm}$ 左右的球形, 覆盖于表流湿地表层土壤与水面之间, 用小木桩将其固定, 载体的填充 率为 $28 \%$ (填充率: 放人载体的体积占表流湿地系统中污水体积的百分比).

\section{4 模型运行及试验方法}

本次试验的时间为 2011 年 5-7 月. 试验前期, 先在表流湿地表面设置生物膜载体, 经过 $20 \mathrm{~d}$ 生物膜挂 膜成功后开始试验. 试验的运行方式为推流式, 采用了较大的水力负荷, 为 $1.50 \mathrm{~m}^{3} /\left(\mathrm{m}^{2} \cdot \mathrm{d}\right)$. 试验进行过程 中每天上午 $8: 00$ 取样进行检测,每组试验重复进行 3 次.

试验期间定期在模型的出水口取水样进行水质 $\mathrm{COD}_{\mathrm{Cr}} 、 \mathrm{TN} 、 \mathrm{TP} 、 \mathrm{SS}$ 指标的测定, 参照《水和废水监测分 析方法》(第 4 版) 进行测定 ${ }^{[15]}$. 采用磷脂脂肪酸法 (PLFA) ${ }^{[16-17]}$ 分析试验稳定运行后载体及湿地系统表层 $0 \sim 5 \mathrm{~cm}$ 处土壤中的微生物.

\section{2 结果}

\section{1 对 $\mathrm{COD}_{\mathrm{Cr}}$ 的去除效果}

设置载体后, 低污染负荷下系统出水 $\mathrm{COD}_{\mathrm{Cr}}$ 浓度由 $59.15 \mathrm{mg} / \mathrm{L}$ 降至 $45.47 \mathrm{mg} / \mathrm{L}$, 去除率提高 $7.72 \%$ (表 1); 高污染负荷下, $\mathrm{COD}_{\mathrm{Cr}}$ 由 $219.15 \mathrm{mg} / \mathrm{L}$ 降至 $87.47 \mathrm{mg} / \mathrm{L}$, 去除率提高 $19.12 \%$, 出水 $\mathrm{COD}_{\mathrm{Cr}}$ 由原来未达 到污水排放标准提升至符合 II 级污水排放标准 $\left(\mathrm{COD}_{\mathrm{Cr}}\right.$ : $\left.100 \mathrm{mg} / \mathrm{L}\right)$.

\section{2 对 TN 的去除效果}

设置生物膜载体后, 系统的出水 $\mathrm{TN}$ 浓度明显变低, 高浓度试验组的净化效果更为显著. 载体设置后, 低 污染负荷下, 系统出水 TN 浓度由 $2.58 \mathrm{mg} / \mathrm{L}$ 降至 $1.96 \mathrm{mg} / \mathrm{L}$, 去除率提高 $3.37 \%$; 载体设置后, 高污染负荷 下, 系统出水 $\mathrm{TN}$ 浓度由 $9.58 \mathrm{mg} / \mathrm{L}$ 降至 $3.96 \mathrm{mg} / \mathrm{L}$, 去除率提高 $21.64 \%$ (表 1). 这表明生物膜载体设置显 著提高了湿地系统除氮能力.

\section{3 对 TP 的去除效果}

载体设置后, 低浓度组系统的出水 TP 浓度由 $0.85 \mathrm{mg} / \mathrm{L}$ 降至 $0.76 \mathrm{mg} / \mathrm{L}$, 去除率提高 $4.16 \%$ (表 1); 高 浓度污水组, 系统 TP 的出水浓度由 $3.52 \mathrm{mg} / \mathrm{L}$ 降至 $1.89 \mathrm{mg} / \mathrm{L}$, 去除率提高 $16.24 \%$, 高浓度污水组的效果 更为明显. 高浓度污水组的试验中, 出水 TP 由原来相当于污水排放 III 级标准提升至 II 级标准 ${ }^{[18]}$.

\section{4 对 SS 的去除效果}

载体设置后, 高污水浓度情况下较大地提高了系统对 SS 的净化效果, 与之相比, 低污水浓度时效果不 明显. 两种情况下,系统对 SS 的净化效果都较好,去除率均可达到 90\% 以上(表 1).

$\mathrm{COD}_{\mathrm{Cr}} 、 \mathrm{TN} 、 \mathrm{TP} 、 \mathrm{SS}$ 的变化均表明: 载体的引人大大降低了湿地系统各水质指标的出水浓度, 在高污染负 荷情况下, 效果更为显著. 与利用生物法-人工湿地组合工艺处理小城镇混合污水 ${ }^{[19]}$ 的处理效果接近, 且未 增加表流人工湿地的占地面积.

表 1 不同污染负荷下湿地系统对各指标的去除率 $(\%)$

Tab. $1 \mathrm{COD}_{\mathrm{Cr}}$, TN, TP, SS removal rates under different pollution loads

\begin{tabular}{rcrrrr}
\hline & & \multicolumn{1}{c}{$\mathrm{COD}_{\mathrm{Cr}}$} & \multicolumn{1}{c}{$\mathrm{TN}$} & \multicolumn{1}{c}{$\mathrm{TP}$} & \multicolumn{1}{c}{$\mathrm{SS}$} \\
\hline \multirow{2}{*}{ 低浓度组 } & 无载体 & $74.48 \pm 0.75$ & $82.66 \pm 0.52$ & $67.80 \pm 2.04$ & $93.06 \pm 0.02$ \\
& 有载体 & $82.20 \pm 0.50$ & $86.03 \pm 0.33$ & $71.96 \pm 2.08$ & $93.71 \pm 0.03$ \\
& 增加量 & $7.72 \pm 0.25$ & $3.37 \pm 0.19$ & $4.16 \pm 0.07$ & $0.65 \pm 0.01$ \\
高浓度组 & 无载体 & $67.30 \pm 0.45$ & $63.36 \pm 0.51$ & $61.00 \pm 1.24$ & $90.73 \pm 0.47$ \\
& 有载体 & $86.42 \pm 0.16$ & $85.00 \pm 0.05$ & $77.25 \pm 0.53$ & $95.92 \pm 0.19$ \\
& 增加量 & $19.12 \pm 0.29$ & $21.64 \pm 0.46$ & $16.24 \pm 0.71$ & $5.18 \pm 0.28$ \\
\hline
\end{tabular}

\section{3 生物膜设置前后表流湿地系统中微生物系统的变化}

表流湿地中微生物主要分布在湿地土壤, 部分以生物膜的方式附着于湿地植物茎杆表面. 为了分析设 置生物膜载体以后湿地微生物系统的变化, 对设置前后湿地表层 $0 \sim 5.0 \mathrm{~cm}$ 范围土壤中微生物的种类和数 
量进行实测, 同时也通过磷脂脂肪酸法对生物膜载体上的微生物种类和数量进行测定 (表 2). 细菌通过甘油 相连的饱和或单不饱和脂肪酸来实测, 如: $12: 0 \sim 20: 0$ 等; 好氧菌以单不饱和脂肪酸来实测, 包括 $114: 0$ 、 a14 :0、15:0 20H、15:0 30H、a15:0、15:0、16:lw7、16:lw7t、16:lw9、16:lw9t、18:lw7、18:1w7t 等; 厌氧菌包括 cy17:0、cy19:0 等; 放线菌包括 10Me16:0、10Me17:0、10Me18:0 等; 硫酸盐还原菌包括 10Me 16:0、117:1w7、 $17: \operatorname{lw} 6$ 等; 真菌包括 $18: 3 \omega 6 \mathrm{c}(6,9,12) 、 18: \operatorname{lw} 9 、 18: 2 \mathrm{w} 6 、 18: 3 \mathrm{w} 6 、 18: 3 \mathrm{w} 3 、 18: 2 \mathrm{w} 9 、 18: 1 \mathrm{w} 9 \mathrm{t}$ 等; 原生动物包 括 $20: 3 w 6 、 20: 4 \omega 6,9,12,15 c$ 等; 嗜热解氢杆菌包括 $18: 0$ 等.

表 2 湿地系统土壤及载体的微生物种类及数量 $(\mathrm{nmol} / \mathrm{g})$

Tab. 2 Numbers and kinds of microorganism in wetland soil and biofilm carrier

\begin{tabular}{|c|c|c|c|c|c|c|}
\hline \multirow{2}{*}{ 微生物 } & \multicolumn{2}{|c|}{ 土壤 (低浓度) } & \multicolumn{2}{|c|}{ 土壤 (高浓度) } & \multicolumn{2}{|c|}{ 载体 } \\
\hline & 无载体 & 有载体 & 无载体 & 有载体 & 低浓度 & 高浓度 \\
\hline 细菌 & 434.802 & 485.489 & 833.769 & 761.681 & 1044.397 & 1884.956 \\
\hline 好氧菌 & 30.558 & 35.593 & 104.986 & 85.052 & 424.804 & 634.481 \\
\hline 厌氧菌 & 3.217 & 6.310 & 8.829 & 8.779 & 43.676 & 24.414 \\
\hline 放线菌 & 1.325 & 8.410 & 26.309 & 23.262 & - & - \\
\hline 硫酸盐还原菌 & 4.535 & 0.467 & - & 17.757 & 3.543 & 1.003 \\
\hline 真菌 & 30.294 & 103.399 & 140.292 & 149.892 & 211.706 & 429.625 \\
\hline 原生动物 & 3.789 & 3.573 & 4. 102 & 5.245 & - & - \\
\hline 嗜热解氢杆菌 & 132.158 & 0.306 & 200.124 & 173.464 & 65.539 & 165.575 \\
\hline 总计 & 640.678 & 643.547 & 1318.411 & 1225.132 & 1793.664 & 3140.054 \\
\hline
\end{tabular}

湿地土壤中以细菌和真菌居多, 嗜热解氢杆菌也比较多见. 随着试验污水的浓度提高, 高浓度组的微生 物数量有所增加, 是原来微生物量的 1 倍左右, 微生物的种群变化也不明显. 设置生物膜以后湿地土壤中微 生物的种群变化不大. 就数量而言, 在低浓度污水的条件下似乎稍有增加的趋势, 相反, 在高浓度污水运行 时, 湿地土壤中的微生物却略有减少. 总体而言, 虽然湿地表面增加了一些生物膜载体, 但对于土壤原有的 微生物在种群和数量上的影响不大.

相对于湿地土壤而言, 设置的载体上附着了大量的微生物. 单位质量载体上附着的微生物数量超过湿 地土壤的 2 倍以上. 从种群上看, 细菌、好氧菌和真菌的增加尤为显著, 放线菌和原生动物则在载体上难以 附着,这一部分的微生物是由于生物膜的设置而产生的增量.

\section{4 讨论与结论}

1) 本次试验设置生物膜以后的处理效果具有两方面的特点:一是考虑到农村用地面积问题使用了较大 的水力负荷 $\left(1.50 \mathrm{~m}^{3} /\left(\mathrm{m}^{2} \cdot \mathrm{d}\right)\right)$; 二是试验污水中 $\mathrm{SS}$ 的含量比实际生活污水要略低. 在这两个试验条件下, 湿地的净化效率受到了一定的限制, 截留、微生物转化等作用在很短的滞留时间内难以发挥, 高、低浓度试 验组各指标的去除率(SS 除外)均在 60\% 左右. 但通过载体设置以后, 各指标的去除率能够上升到 $80 \%$ 左 右, 说明载体设置以后系统对污染物的净化能力显著提高.

2) 系统净化能力提高的机理可以从微生物数量的增加以及微生物与水中污染物接触机会的增多两个 方面解释. 从微生物分析结果来看, 载体的设置没有对原来湿地微生物系统造成大的影响, 进而增加了系统 中的微生物密度. 这些微生物的作用, 是去除率上升 $20 \%$ 的主要原因.

3) 考虑到农村的具体情况, 本次试验未采用工业用的生物膜载体, 而是使用农村常见的农业废弃 物——玉米皮经过简单加工后进行使用. 从生物膜的附着效果来看, 基本上起到了载体的效果. 而且在进行 试验的半年期间, 由玉米皮加工而成的载体没有出现腐烂、破碎的迹象. 可以认为, 利用玉米皮加工成的生 物膜载体具有一定的可使用性.

\section{5 参考文献}

[ 1 ] Zhang DQ, Gersberg RM, Keat TS. Constructed wetlands in China. Ecol Eng, 2009,35(10):1367-1378. 
［2］2010 中国环境状况公报. 中华人民共和国环境保护部,2011.

[ 3 ] Vymazal J. The use constructed wetlands with horizontal sub-surface flow for various types of wastewater. Ecol Eng, 2009, $35: 1-17$.

[ 4 ] Blazejewski R, Murat-Blazejewska S. Soil clogging phenomena in constructed wetlands with subsurface flow. Wat Sci Tech, 1997,35 : 183-188.

[ 5 ] Hua GF, Zhu W, Zhang YH. A conceptual approach based on suspended solids to estimate clogging time in constructed wetlands. Journal of Environmental Science and Health, 2010,45: 1519-1525.

[6] Chescheir GM, Skaggs RW, Gilliam JW. Evaluation of wetland buffer areas for treatment of pumped agricultural drainage water. Transactions of the American Society of Agricultural Engineers, 1992 ,35:175-182.

[ 7 ] Braskerud BC. Factors affecting nitrogen retention in small constructed wetlands treating agricultural non-point source pollution. Ecol Eng, 2002,18:351-370.

[8] 田卫.表面流人工湿地净化污水的应用研究 [学位论文]. 长春:吉林大学, 2004:5.

[9] 陈俊敏, 贾滨洋, 付永胜. 表面流人工湿地用于农家乐旅游设施污水处理的试验研究. 农科环境科学学报,2006,25 (增刊) : 191-193.

[10 ] Reddy KR, D’ Angelo EM. Soil processes regulating water quality in wetlands. In: Mitsch WJ ed. Global wetlands — Old world and new. New York: Elsevier, 1994:309-324.

[11] 张显芸. 表流湿地净化面源污染的机理研究 [学位论文]. 南京:河海大学, 2009:5.

[12] Tao WD, Hall KJ, Duff SJB. Microbial biomass and heterotrophic production of surface flow mesocosm wetlands treating woodwaste leachate: Responses to hydraulic and organic loading and relations with mass reduction. Ecological Engineering, $2007, \mathbf{3 1}: 132-139$.

[13] 付融冰, 杨海真, 顾国维等. 人工湿地基质微生物状况与净化效果相关分析. 环境科学研究, 2005,18(6):44-49.

[14] Iamchaturapatr J, Yi SW, Rhee JS. Nutrient removals by 21 aquatic plants for vertical free surface-flow ( VFS) constructed wetland. Ecological Engineering, $2007,29: 287-293$.

[15] 国家环境保护总局《水和废水监测分析方法》编委会. 水和废水监测分析方法: 第 4 版. 北京: 中国环境科学出版 社, 2002 .

[16] Zelles L, Bai QY. Fractionation of fatty acids derived from soil lipids by solid phase extraction and their quantitative analysis by GC-MS. Soil Biol Biochem, $1993,25: 495-507$.

[17] Guckert JB, Hood MA, White DC. Phospholipid ester-linked fatty acid profile changes during nutrient deprivation of Vibrio cholerae: increases in the trans/cis ratio and proportions of cyclopropyl fatty acids. Appl Environ Microbiol, 1986,52: $794-801$.

[18］ GB/T 18918-2002. 城镇污水处理厂污染物排放标准, 2002.

[19] 卢 建,杨 扬, 尹振娟等. 生物法-人工湿地组合工艺处理小城镇混合污水研究. 环境工程学报, 2010,4 (6): $1262-1266$. 